\title{
Paternally inherited microdeletion at $15 q 11.2$ confirms a significant role for the SNORD116 C/D box snoRNA cluster in Prader-Willi syndrome
}

\author{
Angela L Duker ${ }^{1}$, Blake C Ballif ${ }^{2}$, Erawati V Bawle ${ }^{1}$, Richard E Person ${ }^{3}$, Sangeetha Mahadevan ${ }^{3}$, \\ Sarah Alliman ${ }^{2}$, Regina Thompson ${ }^{2}$, Ryan Traylor ${ }^{2}$, Bassem A Bejjani $^{2}$, Lisa G Shaffer ${ }^{2}$, Jill A Rosenfeld ${ }^{2}$, \\ Allen N Lamb ${ }^{2}$ and Trilochan Sahoo ${ }^{\star, 2}$
}

Prader-Willi syndrome (PWS) is a neurobehavioral disorder manifested by infantile hypotonia and feeding difficulties in infancy, followed by morbid obesity secondary to hyperphagia. It is caused by deficiency of paternally expressed transcript(s) within the human chromosome region 15q11.2. PWS patients harboring balanced chromosomal translocations with breakpoints within small nuclear ribonucleoprotein polypeptide $\mathbf{N}$ (SNRPN) have provided indirect evidence for a role for the imprinted C/D box containing small nucleolar RNA (snoRNA) genes encoded downstream of SNRPN. In addition, recently published data provide strong evidence in support of a role for the snoRNA SNORD116 cluster (HBII-85) in PWS etiology. In this study, we performed detailed phenotypic, cytogenetic, and molecular analyses including chromosome analysis, array comparative genomic hybridization (array CGH), expression studies, and single-nucleotide polymorphism (SNP) genotyping for parent-of-origin determination of the 15q11.2 microdeletion on an 11-year-old child expressing the major components of the PWS phenotype. This child had an $\sim 236.29 \mathrm{~kb}$ microdeletion at 15q11.2 within the larger Prader-Willi/Angelman syndrome critical region that included the SNORD116 cluster of snoRNAs. Analysis of SNP genotypes in proband and mother provided evidence in support of the deletion being on the paternal chromosome 15. This child also met most of the major PWS diagnostic criteria including infantile hypotonia, early-onset morbid obesity, and hypogonadism. Identification and characterization of this case provide unequivocal evidence for a critical role for the SNORD116 snoRNA molecules in PWS pathogenesis. Array CGH testing for genomic copy-number changes in cases with complex phenotypes is proving to be invaluable in detecting novel alterations and enabling better genotype-phenotype correlations.

European Journal of Human Genetics (2010) 18, 1196-1201; doi:10.1038/ejhg.2010.102; published online 30 June 2010

Keywords: Prader-Willi syndrome; snoRNA; microdeletion; array CGH

\section{INTRODUCTION}

Prader-Willi syndrome (PWS; MIM 176270) is a neurobehavioral disorder caused by the lack of paternal expression of imprinted genes in the human chromosome region 15q11.2q13. PWS manifests as infantile hypotonia, genital hypoplasia, and neonatal feeding difficulties, followed by hyperphagia leading to profound obesity in early childhood and into adulthood. ${ }^{1,2}$ Large interstitial deletions of $\sim 6-6.8 \mathrm{Mb}$ at $15 \mathrm{q} 11.2 \mathrm{q} 13$ of paternal origin are the cause in over $70 \%$ of cases. The majority of the remaining cases have maternal uniparental disomy (UPD) 15, and a small percentage have imprinting defects. Deletions at $15 \mathrm{q} 11.2 \mathrm{q} 13$ of maternal origin result in Angelman syndrome (MIM 105830). Identification and characterization of the recurrent deletion breakpoints have revealed low-copy repeat-mediated nonallelic homologous recombination as the unifying mechanism for the common deletions and duplications across this interval. ${ }^{3,4}$

A number of paternally expressed genes mapping within this critical interval have been implicated in PWS pathogenesis. The small nuclear ribonucleoprotein polypeptide $\mathrm{N}(S N R P N)$ gene encodes a bicistronic (SNURF and SmN) transcript and was initially considered a strong
PWS candidate gene, but subsequent evidence from patients with balanced translocations have excluded it as a primary candidate., Located within the downstream introns of SNRPN are clusters of C/D box-containing small nucleolar RNA (snoRNA) genes. The expression of these snoRNAs is under control of the SNRPN promoter, is allelespecific with highest paternal expression in the brain, and has been speculated to function toward guiding $2^{\prime}$-O-ribose methylation of ribosomal RNA or small nuclear RNA. ${ }^{7-9}$

Chromosomal rearrangements often provide important clues toward identifying loci that contribute to a disease phenotype. Six cases with PWS or PWS-like phenotype harboring balanced reciprocal translocations with breaks within the SNRPN locus have been reported. ${ }^{10}$ In addition, there are now two reports of microdeletions immediately distal to or overlapping the terminal exons of SNRPN that lead to complete loss of the SNORD116 snoRNA cluster in both cases and a substantial segment of the SNORD115 (HBII-52) cluster in one. ${ }^{9,11}$

Here we report a microdeletion within 15q11.2 leading to complete loss of the SNORD116 snoRNA cluster in a child manifesting the

\footnotetext{
${ }^{1}$ Division of Genetic and Metabolic Disorders, Children's Hospital of Michigan, Detroit, MI, USA; ${ }^{2}$ Signature Genomic Laboratories, Spokane, WA, USA; ${ }^{3}$ Department of Molecular and Human Genetics, Baylor College of Medicine, Houston, TX, USA

*Correspondence: Dr T Sahoo, Signature Genomic Laboratories, 2820 North Astor, Spokane, WA 99207, USA. Tel: +1 509 474 6840 ; Fax: +1 509 474 6839;

E-mail: sahoo@signaturegenomics.com
}

Received 10 February 2010; revised 20 April 2010; accepted 2 June 2010; published online 30 June 2010 
major features of PWS. This interstitial deletion of $\sim 236 \mathrm{~kb}$, involving the $3^{\prime}$ end of $S N R P N$, does not negatively impact the physical integrity or expression of other imprinted genes in its vicinity. A combination of high-resolution deletion mapping, expression studies, and phenotypic characterization provide, in conjunction with data from the two recently published cases, unequivocal evidence for a critical role for this cluster of snoRNAs in the molecular etiology of PWS. This also provides significant reason to investigate the functional roles of snoRNAs in gene expression and function, especially disruption of the SNORD116 cluster at 15q11.2 in early-onset morbid obesity patients.

\section{PATIENTS AND METHODS}

\section{Patient report}

The patient is an 11-year-old African-American boy with morbid obesity, developmental delay, medical history of hypotonia, and feeding difficulties. The patient was born at full term (by date) without complications. His birth weight was $3020 \mathrm{~g}$, length $53 \mathrm{~cm}$, and FOC $32.6 \mathrm{~cm}$, all within normal range. There were no pregnancy-related medical problems. The neonatal period was significant for weak sucking skill requiring nasogastric tube feeding for the first 4 weeks of life, weak cry, and undescended testis. Hypotonia and weak sucking was significant from birth. Excessive weight gain was apparent by 6 months of age ( $10.8 \mathrm{~kg}$ by 7 months and $13 \mathrm{~kg}$ by 9 months).

At 2 years of age his examination was notable for very few obvious dysmorphic features: height and head circumference both at approximately 90th centile, BMI of $33 \mathrm{~kg} / \mathrm{m}^{2}$, inverted-V-shaped upper lip, and small testes following an orchiopexy. He also had a history of seizures that have now resolved.

Reevaluation at age 11 years revealed generalized obesity (Figure 1), height at 10-25th centile, head circumference at the 98th centile, weight $94 \mathrm{~kg}$ with a BMI of $50 \mathrm{~kg} / \mathrm{m}^{2}$, inverted-V-shaped upper lip, dark black hair consistent with his African-American ancestry, middle finger length at 10th centile, palm length 90th centile, complete horizontal creases on both palms, small testes, and penis length between 3rd and 10th centile. He has generalized mild hypotonia and can stand and walk only with support and ambulates with the help of a rolling walker. He fatigues with short periods of crawling or walking. He is hyperphagic and engages in food foraging behavior, but his ability to do so is limited because of the motor deficit. In addition, he has a history of sleep apnea. He shows thick and viscous saliva and has esotropia. Skin picking was not reported by parents nor observed at examination. He apparently has a high pain threshold.

He has moderate mental retardation, with some speech articulation defects. He apparently is argumentative, and has some obsessive-compulsive behavior. $\mathrm{He}$ is enrolled in a class for cognitively impaired. He is independent in feeding and toileting. He has difficulty with planning of motor activities and apraxia

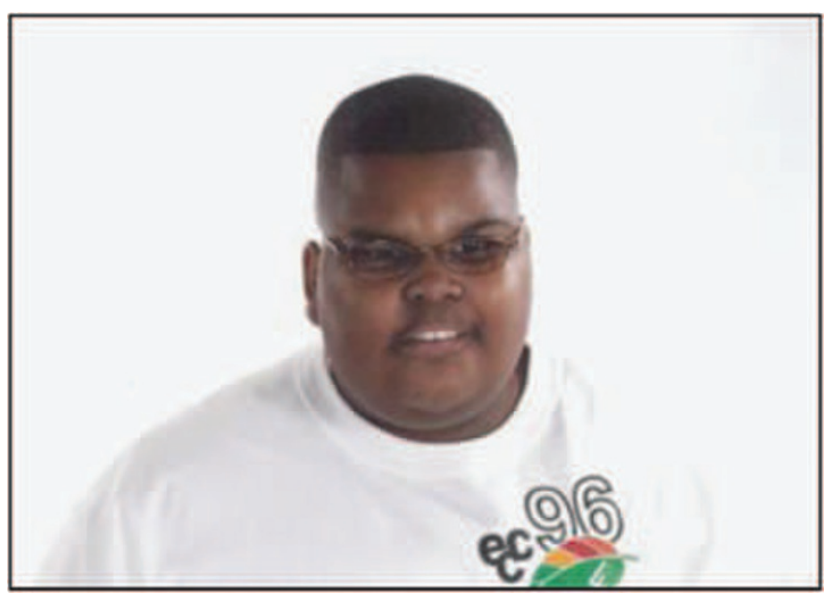

Figure 1 Profile of patient at age 11 years. Note morbid obesity. for writing. His short-term memory is also poor. Speech and language evaluation by Peabody Picture Vocabulary Test-III showed age-equivalent skills of 4 years for receptive vocabulary. He can say six-word sentences but has some trouble in articulation. He is receiving physical, occupational, and speech therapy in school. He plays some with peers but relates better with adults and reportedly loves and excels at solving puzzles.

Family history shows he has a maternal half brother and five paternal half siblings who have no history of mental retardation, morbid obesity, or congenital anomalies. Likewise his mother and her immediate relatives have no history of mental retardation, infant deaths, congenital anomalies, morbid obesity, or genetic syndromes. His father and paternal grandparents are obese, but there is no known paternal family history of mental retardation, hypotonia, or genetic syndromes. There is no history of parental consanguinity. His maternal and paternal ethnicity is African American.

Based on the phenotype, a clinical diagnosis of PWS was not confirmed by either fluorescence in situ hybridization (FISH) for deletion of chromosome 15q11.2 or methylation analysis for UPD and imprinting defects. Routine chromosome analysis and subtelomere FISH were normal. Serum very long chain fatty acids, urine organic acid analysis, serum CPK, and muscle biopsy were nondiagnostic.

\section{Molecular and microarray-based comparative genomic hybridization analysis}

Oligonucleotide-based array comparative genomic hybridization (array CGH) analysis was initially performed using a $135 \mathrm{k}$-feature whole-genome microarray (SignatureChip OS2.0 manufactured for Signature Genomic Laboratories (Spokane, WA, USA) by Roche NimbleGen, Madison, WI, USA; based on UCSC 2006 hg18 assembly). This array targets $>200$ known genetic disorders including all currently known microdeletion-microduplication syndrome regions and $>675$ functionally significant genes in addition to all subtelomeric and pericentromeric regions of the genome. The genomic backbone of this array includes one probe every $35 \mathrm{~kb}$ with targeted regions consisting of one probe every $10 \mathrm{~kb}$. Genomic DNA was extracted from peripheral blood using a Qiagen M48 Biorobot automated DNA extraction system (Qiagen Inc, Valencia, CA, USA). Purified genomic DNA was then labeled with Cyanine dyes Cy3 or Cy5 using a Roche NimbleGen DNA labeling kit. Array hybridization and washing were performed as specified by the manufacturer (Roche NimbleGen). Arrays were scanned using an Axon 4000B scanner (Molecular Devices, Sunnyvale, CA, USA) and analyzed using GenePix 6.1 (Molecular Devices), DNA Analytics 4.0 (Agilent Technologies, Santa Clara, CA, USA) and NimbleScan 2.5 (Roche NimbleGen). Results were then displayed using custom oligonucleotide array CGH analysis software (Genoglyphix; Signature Genomic Laboratories).

FISH was performed to confirm and visualize the deletion using BAC clone CTD-2283B2 from the deleted region using previously published methods. ${ }^{12}$

\section{Deletion size and breakpoint analysis}

High-resolution microarray analysis was performed using the NimbleGen Human Copy Number Variation $2.1 \mathrm{M}$ array, interrogating with 2.1 million probes at an average spacing of $\sim 1.2 \mathrm{~kb}$, to delineate the deletion size further.

\section{Expression studies}

Total RNA was extracted from whole blood (patient) and transformed lymphoblast cell lines (for two normal control samples) and treated with DNaseI before RT-PCR (Qiagen miRNeasy kit, Qiagen Inc). RT-PCR was performed using primers that were designed within and across exons for SNRPN (exon 1-3), SNORD116 and UBE3A following methods previously described. GAPDH was used as the internal control. ${ }^{9,10,13}$ SNRPN (exon 1-3), and UBE3A (exon 15 and 16) were chosen to rule out any effect that the deleted segment might have had on the expression of upstream and downstream elements.

\section{SNP genotyping to determine parent of origin}

The Affymetrix Genome-Wide Human SNP Array 6.0, featuring more than 906600 single-nucleotide polymorphisms (SNPs) and more than 946000 probes for the detection of copy-number variation, was used for genotyping as well as estimating the deletion size (Affymetrix, Santa Clara, CA, USA). 


\section{RESULTS}

Array CGH analysis using a 135k feature oligonucleotide microarray revealed a copy-number loss encompassing a segment of $\sim 219 \mathrm{~kb}$ at 15q11.2. FISH confirmed an apparent interstitial deletion at $15 q 11.2$ [arr 15q11.2(22 782 259-23000 927)x1; ish del(15)(q11.2q11.2)(CTD2283B2-)] (Figures 2a and b). Maternal FISH analysis was normal (paternal sample was unavailable for testing). Six additional CNVs were identified (104 kb gain at 6p25.3; $92 \mathrm{~kb}$ loss at 8p11.23; $90 \mathrm{~kb}$ loss at $17 \mathrm{q} 21.31 ; 16 \mathrm{~kb}$ loss at $22 \mathrm{q} 11.22$ (distal to DiGeorge syndrome critical interval); $102 \mathrm{~kb}$ loss at Xp22.33; $103 \mathrm{~kb}$ loss at Xq28), all of which are considered benign variants as they are seen at a high frequency in the control population (Database of Genomic Variants; http://projects.tcag.ca/variation), may not be overlapping known genes, and are identified in control samples at Signature Genomic Laboratories.

Deletion size analysis using the NimbleGen $2.1 \mathrm{M}$-feature microarray indicated that the deletion boundary was between positions 22774847 and 23007783 (a deletion of $\sim 233 \mathrm{~kb}$ based on HG18 NCBI Build 36.1, March 2006). Sequencing of a junction fragment of $\sim 4 \mathrm{~kb}$ obtained by long-range PCR revealed breakpoints at position 22774712 (proximal) and 23011007 (distal) with the insertion of a single base $(\mathrm{G})$ at the breakpoint. The deleted segment was now determined to be exactly $236295 \mathrm{bp}$. The proximal breakpoint overlaps the $3^{\prime}$ end of SNRPN (partially overlapping the terminal coding exon 10), and the telomeric breakpoint is between snoRNA box
SNORD115-24 and SNORD115-25. The deleted segment therefore includes the noncoding transcripts C/D box snoRNAs SNORD107, 64, 108, 109, SNORD116 cluster 1-29, and SNORD115 clusters 1-24 (Figures $2 \mathrm{a}$ and $3 \mathrm{a}$ ).

The genomic segment $(5 \mathrm{~kb})$ immediately downstream of the breakpoint does not contain any significant structural elements that could have contributed to the deletion. There were no segmental duplications immediately flanking the breakpoints, suggesting a random breakage and nonhomologous end joining as the most likely mechanism for the formation of this deletion. The downstream segment contains a number of small CNVs reported in the Database of Genomic Variants (http://projects.tcag.ca/). These CNVs overlapped with the SNORD115 cluster of snoRNAs.

RT-PCR revealed normal expression of SNRPN and UBE3A with complete loss of SNORD116 expression. Maternally expressed (brainspecific) $U B E 3 A$ was analyzed using only peripheral blood RNA and hence normal expression was observed as expected (Figure 3b). SNORD115 snoRNA expression has been shown to be absent in peripheral tissues and hence no expression analysis was performed. ${ }^{8}$

There was complete agreement between the deletion size estimated by the Affymetrix Genome-Wide Human SNP Array 6.0 and that determined by the NimbleGen $2.1 \mathrm{M}$ probe CNV array. Genotype analysis for all SNPs within the deleted segment at 15q11.2 (77 SNPs present; 76 unambiguously called) revealed, as expected, a block of apparent loss of heterozygosity in the deleted interval. Comparison of

a
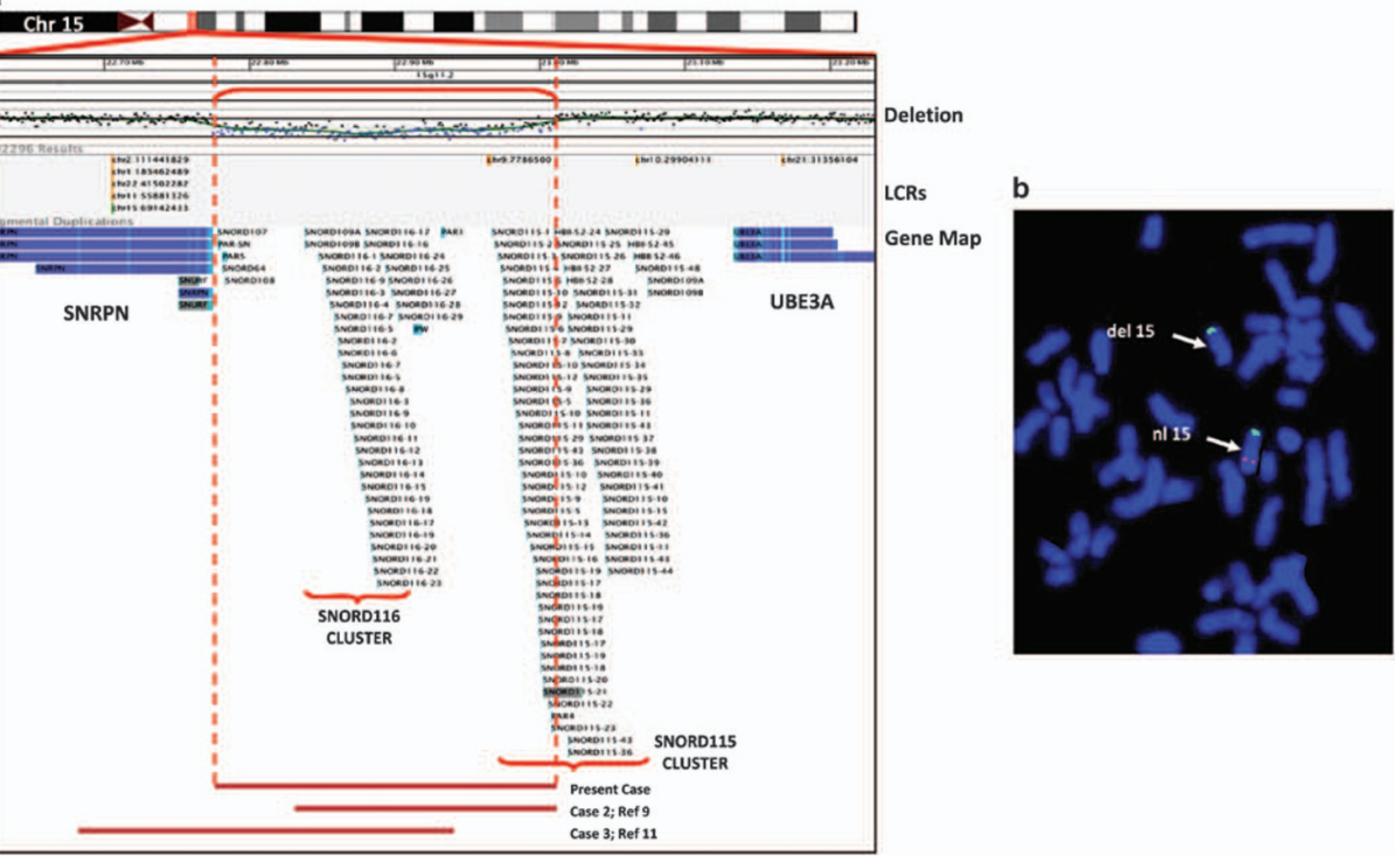

Figure 2 Characterization of microdeletion at 15q11.2 by microarray and FISH analyses. (a) Array CGH data (Roche NimbleGen 2.1 million CNV array) for proximal 15q11.2, and between SNRPN and UBE3A genes, showing loss in copy number for a segment between $22774847-23007783$ (red bracket and vertical interrupted red lines). Tracks below the copy-number plot show distribution of segmental duplications followed by the track highlighting the gene content within interval. The SNORD116 and SNORD115 snoRNA clusters are highlighted by horizontal (red) brackets at the bottom of track. Results were visualized using Genoglyphix software (Signature Genomic Laboratories). The three reported deletions (including present case) shown at bottom of panel depicting relative genomic position and size. (b) Analysis by FISH with clone CTD-2283B2 (red signal) confirmed interstitial deletion at 15q11.2. 
a

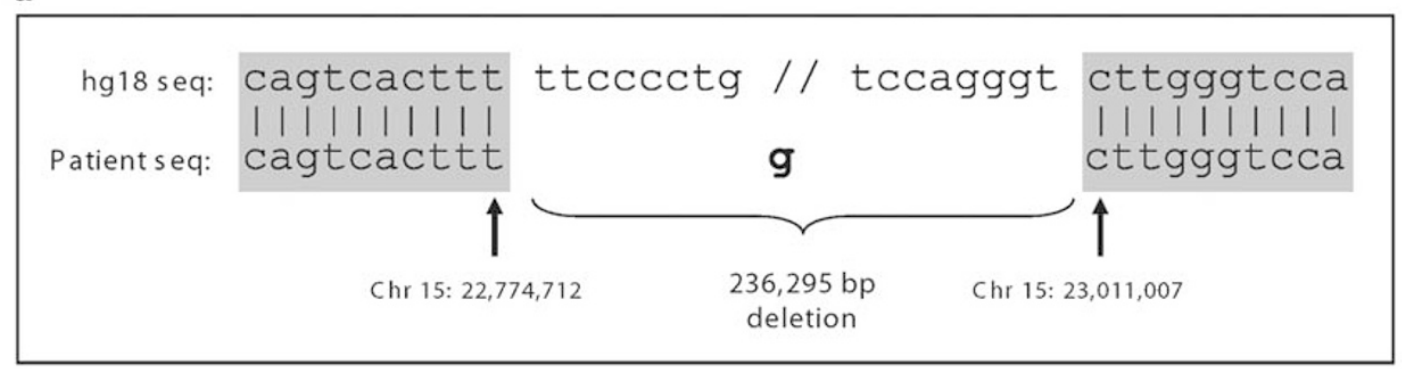

b

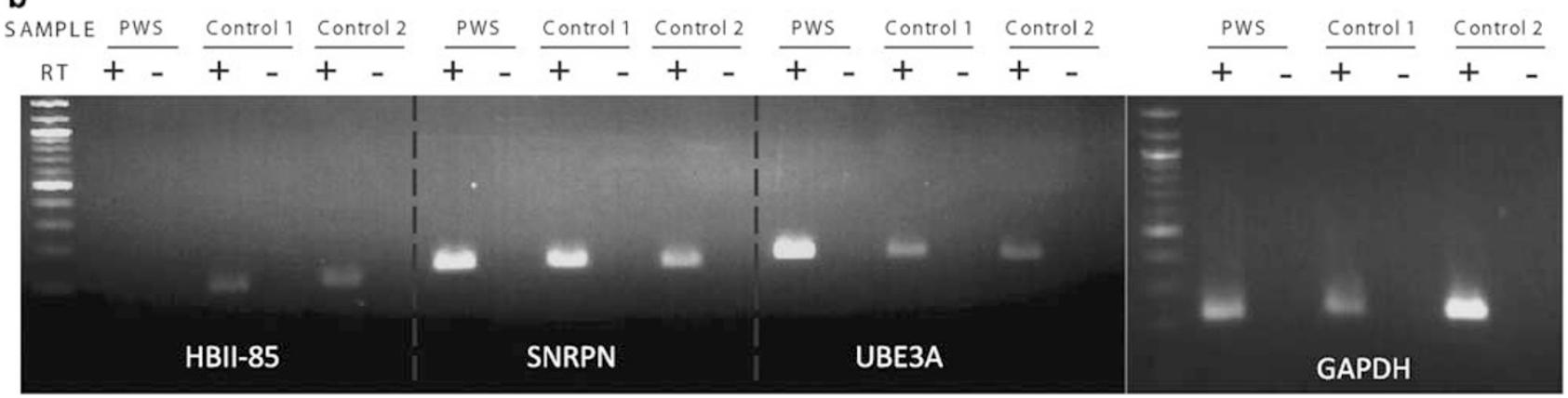

Figure 3 Breakpoint sequence and expression analysis. (a) Sequence analysis of $\sim 4 \mathrm{~kb}$ junction fragment revealed a $236295 \mathrm{bp}$ deletion. Insertion of a single base was also identified at the breakpoint. (b) Expression analysis by RT-PCR of whole blood (patient) or lymphoblast cell (controls) RNA for SNRPN, snoRNA clusters SNORD116, and UBE3A. GAPDH was used as internal RT-PCR control. (RT+: with reverse transcriptase; RT-: without reverse transcriptase). Patient (PWS) was negative for PCR product in RT+ lane for SNORD116 but positive for PCR product for all other tested exons.

the genotyping calls for the 76 unambiguous SNPs in the deleted interval with those of the mother are consistent with inheritance of only maternal alleles, indicative of a hemizygous deletion on paternal chromosome 15 (Supplementary Table S1).

\section{DISCUSSION}

We describe here a patient with physical findings and clinical course consistent with PWS. The patient exhibits many of the major diagnostic criteria that include neonatal and infantile hypotonia, feeding difficulties that lasted about the first 4 weeks, followed by excessive weight gain by age 7 months, hyperphagia, hypogonadism, and global developmental delay. In addition, he has some other minor features including behavioral problems, sleep apnea, and speech delay.

The patient, however, lacks some of the facial features that have been described in PWS patients (Figure 1). The clinical features and physical findings of our patient were compared with other patients who have a cytogenetic abnormality that involves this region (Table 1). ${ }^{9,11}$ Negative results from conventional 15q11.2-q13 deletion and methylation analysis carried out in the neonatal period did not confirm a diagnosis of PWS. However, array CGH testing carried out at reevaluation revealed a unique microdeletion restricted to a genomic segment within the PWS-Angelman syndrome critical interval and encompassing a cluster of snoRNAs.

Defining and identifying genes within the PWS deletion region as determinants of the phenotype represent a longstanding problem..$^{5-7,10,14}$ Microdeletions, responsible for $70 \%$ of cases, lead to loss of a number of paternally expressed genes (MKRN3, NECDIN, MAGEL2, SNURF-SNRPN, snoRNAs) within the interval. Mutations have not been identified in any single gene that can be implicated in the PWS phenotype. Balanced reciprocal translocations, though a rare cause of PWS, have provided important clues toward identifying genomic regions and candidate genes responsible for the phenotype.
These rearrangements disrupted genes within the region without affecting its imprinted status. Six reported translocation cases of PWS, with breakpoints within the upstream exons or $3^{\prime} \mathrm{UTR}$ of $S N R P N$, suggest an important role for downstream loci that are under the control of the SNRPN promoter and paternally expressed. ${ }^{5,6,10,15-17}$ With the exclusion of SNRPN as a primary PWS candidate gene, an important role for the noncoding snoRNAs in the PWS phenotype has emerged. These molecules are the only 'functional' elements distributed within downstream introns of SNRPN and are a part of the large transcript initiated from the SNRPN promoter. The snoRNA molecules represent an important subset of noncoding molecules with diverse functions, most recognizable being the $2^{\prime}$-O-methylation of various classes of RNAs. ${ }^{7,8,18,19}$ The multicopy snoRNA cluster SNORD115 (HBII-52) was ruled out as having a major role in PWS from analysis of a microdeletion that included the entire cluster and did not manifest an obvious clinical phenotype when the deletion was on the paternal chromosome. ${ }^{20}$ Interestingly, SNORD115 snoRNA has now been shown to regulate alternative splicing of a serotonin receptor $\left(5-\mathrm{HT}_{2 \mathrm{C}} \mathrm{R}\right)$. This apparently affects the expression of $5-\mathrm{HT}_{2} \mathrm{C}$ isoforms in PWS patients that are different from those expressed in normal individuals, thus impacting a pathway with indirect but considerable influence on the PWS phenotype. $^{21}$ The SNORD116 cluster $(29$ copies) has been implicated from indirect evidence derived from fine mapping of rare balanced translocation cases with PWS. Determination of breakpoints in these translocation carriers with PWS suggests the inclusion of the HBII-85 cluster as a primary component of the minimal critical region.

Two mouse models generated with targeted deletions of the MBII-85 snoRNA cluster have now been characterized. ${ }^{22,23}$ Variable neonatal lethality was observed (variable up to $15 \%$ and background-specific in one model). Interestingly, in both models, the mice showed decreased activity and/or hypotonia at birth and postnatal growth retardation, as 
Table 1 Comparison of clinical features between current patient and previously reported cases with HBII-85 microdeletion 9,11

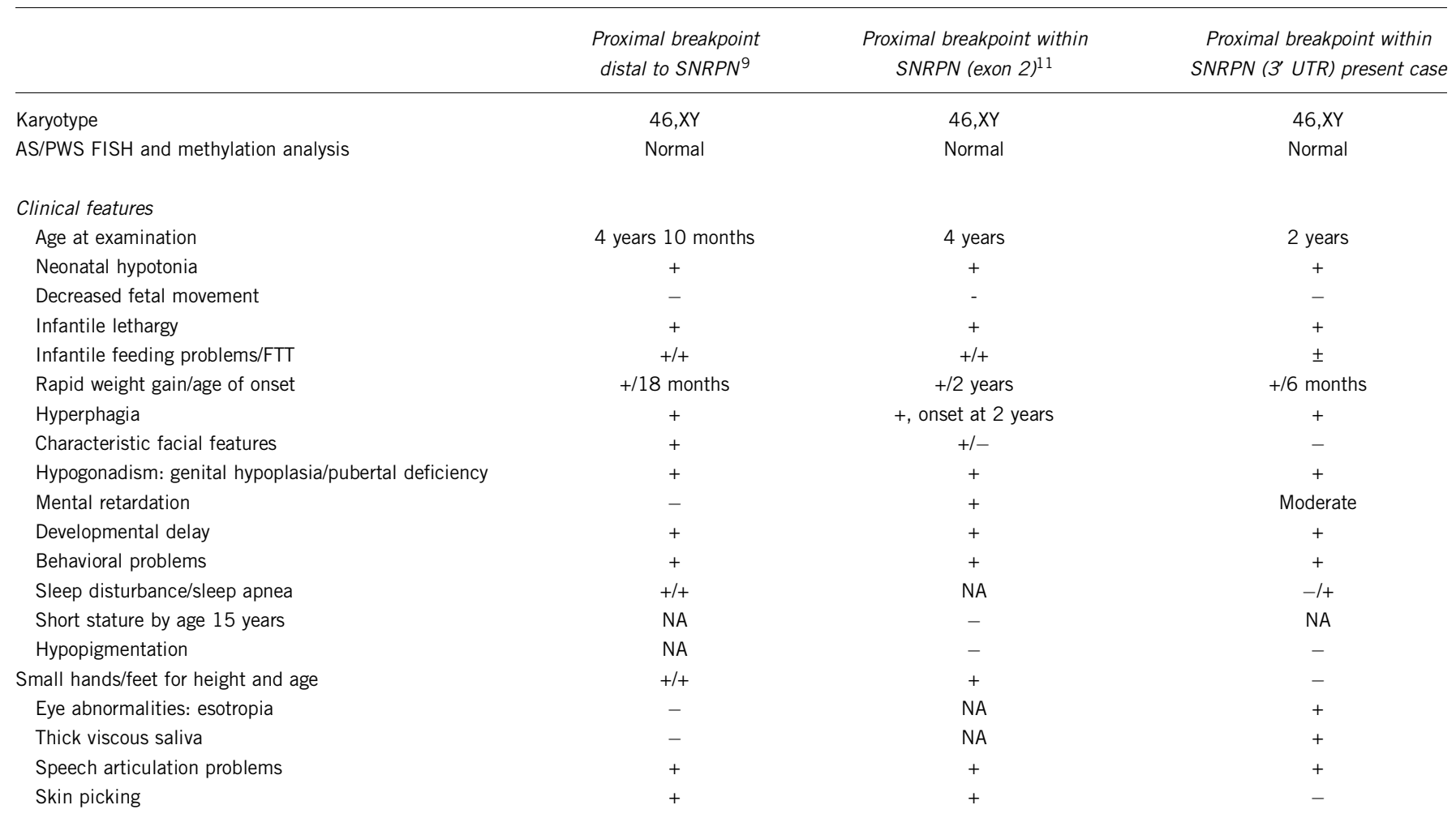

Abbreviations: NA, data not available; FTT, failure to thrive.

has been reported for other PWS mouse models with larger deletions. These mice were not obese or infertile, suggesting species-specific differences. One of the mouse models was described as having deficiency in motor learning, increased anxiety and hyperphagia without obesity. ${ }^{22}$ Overall, these mice recapitulated important components of the PWS phenotype, thus supporting a significant role for snoRNAs in PWS pathogenesis. In addition, studies using human and mice brain tissue suggest that significant decondensation of chromatin occurs at the HB/MBII snoRNA clusters. This effect was neuron specific and dependent upon imprinting and transcriptional status of the snoRNA cluster, suggesting an important role in the neuronal maturation process. $^{24}$

Overwhelming evidence in support of a role for the SNORD116 snoRNA cluster has now been provided by two recently published cases with unique, yet overlapping microdeletions at 15q11.2, leading to loss of the paternal copy of the SNORD116 snoRNAs. ${ }^{9,11}$

In this report, we provide convincing evidence that a small deletion involving SNORD116 snoRNA cluster is sufficient to cause PWS. It is now pertinent to identify RNA substrates for this group of snoRNAs as a step toward understanding its functional role. In addition, it may be prudent to recommend array CGH as a primary diagnostic test for such patients, as conventional cytogenetic analysis has the potential to miss their identification. Conventional methylation analysis and FISH testing, that provides the first line of evidence in support of a clinical diagnosis of PWS, may in these rare cases prove to be negative and preclude an accurate diagnosis.

\section{CONFLICT OF INTEREST}

The authors declare no conflict of interest.

\section{ACKNOWLEDGEMENTS}

We thank the family for its support during this study. We thank Aaron Theisen (Signature Genomic Laboratories) and Dr Jan Bressler (Human Genetics Center, University of Texas Health Science Center, Houston) for critical reading of the paper. Work contributed by REP and SM was carried out in the laboratory of Dr Arthur L Beaudet, Department of Molecular and Human Genetics, Baylor College of Medicine, Houston, TX.

1 Holm VA, Cassidy SB, Butler MG et al: Prader-Willi syndrome: consensus diagnostic criteria. Pediatrics 1993; 91: 398-402.

2 Gunay-Aygun M, Schwartz S, Heeger S et al: The changing purpose of Prader-Willi syndrome clinical diagnostic criteria and proposed revised criteria. Pediatrics 2001; 108: E92.

3 Sahoo T, Bacino CA, German JR et al: Identification of novel deletions of 15q11q13 in Angelman syndrome by array-CGH: molecular characterization and genotype-phenotype correlations. Eur J Hum Genet 2007; 15: 943-949.

4 Makoff AJ, Flomen RH: Detailed analysis of 15q11-q14 sequence corrects errors and gaps in the public access sequence to fully reveal large segmental duplications at breakpoints for Prader-Willi, Angelman, and inv dup(15) syndromes. Genome Biol 2007; 8: R114.

5 Schulze A, Hansen C, Skakkebaek NE et al: Exclusion of SNRPN as a major determinant of Prader-Willi syndrome by a translocation breakpoint. Nat Genet 1996; 12: 452-454.

6 Wirth J, Back E, Huttenhofer A et al: A translocation breakpoint cluster disrupts the newly defined $3^{\prime}$ end of the SNURF-SNRPN transcription unit on chromosome 15. Hum Mol Genet 2001; 10: 201-210.

7 Runte M, Huttenhofer A, Gross S et al: The IC-SNURF-SNRPN transcript serves as a host for multiple small nucleolar RNA species and as an antisense RNA for UBE3A. Hum Mol Genet 2001; 10: 2687-2700.

8 Cavaille J, Buiting K, Kiefmann M et al: Identification of brain-specific and imprinted small nucleolar RNA genes exhibiting an unusual genomic organization. Proc Natl Acad Sci USA 2000; 97: 14311-14316.

9 Sahoo T, del Gaudio D, German JR et al: Prader-Willi phenotype caused by paternal deficiency for the HBII-85 C/D box small nucleolar RNA cluster. Nat Genet 2008; 40: 719-721.

10 Schule B, Albalwi M, Northrop E et al: Molecular breakpoint cloning and gene expression studies of a novel translocation $\mathrm{t}(4 ; 15)(\mathrm{q} 27 ; \mathrm{q} 11.2)$ associated with Prader-Willi syndrome. BMC Med Genet 2005; 6: 18. 
11 de Smith AJ, Purmann C, Walters RG et al: A deletion of the HBII-85 class of small nucleolar RNAs (snoRNAs) is associated with hyperphagia, obesity and hypogonadism. Hum Mol Genet 2009; 18: 3257-3265.

12 Traylor RN, Fan Z, Hudson B et al: Microdeletion of 6q16.1 encompassing EPHA7 in a child with mild neurological abnormalities and dysmorphic features: case report. Mol Cytogenet 2009; 2: 17

13 Rougeulle C, Glatt H, Lalande M: The Angelman syndrome candidate gene, UBE3A/E6AP, is imprinted in brain. Nat Genet 1997; 17: 14-15.

14 Gallagher RC, Pils B, Albalwi M et al: Evidence for the role of PWCR1/HBII-85 C/D box small nucleolar RNAs in Prader-Willi syndrome. Am J Hum Genet 2002; 71: 669-678.

15 Sun Y, Nicholls RD, Butler MG et al: Breakage in the SNRPN locus in a balanced 46,XY,t(15;19) Prader-Willi syndrome patient. Hum Mol Genet 1996; 5: 517-524.

16 Kuslich CD, Kobori JA, Mohapatra G et al: Prader-Willi syndrome is caused by disruption of the SNRPN gene. Am J Hum Genet 1999; 64: 70-76.

17 Conroy JM, Grebe TA, Becker LA et al: Balanced translocation 46,XY,t(2;15) (q37.2;q11.2) associated with atypical Prader-Willi syndrome. Am J Hum Genet 1997; 61: 388-394.
18 de los Santos T, Schweizer J, Rees CA et al: Small evolutionarily conserved RNA, resembling C/D box small nucleolar RNA, is transcribed from PWCR1, a novel imprinted gene in the Prader-Willi deletion region, which is highly expressed in brain. Am J Hum Genet 2000; 67: 1067-1082.

19 Kiss T: Small nucleolar RNAs: an abundant group of noncoding RNAs with diverse cellular functions. Cell 2002; 109: 145-148.

20 Runte M, Varon R, Horn D et al: Exclusion of the C/D box snoRNA gene cluster HBII-52 from a major role in Prader-Willi syndrome. Hum Genet 2005; 116: 228-230.

21 Kishore S, Stamm S: The snoRNA HBII-52 regulates alternative splicing of the serotonin receptor 2C. Science 2006; 311: 230-232.

22 Ding F, Li HH, Zhang S et al: SnoRNA Snord116 (Pwcr1/MBII-85) deletion causes growth deficiency and hyperphagia in mice. PLOS One 2008; 3: e1709.

23 Skryabin BV, Gubar LV, Seeger B et al: Deletion of the MBII-85 snoRNA gene cluster in mice results in postnatal growth retardation. PLoS Genet 2007; 3: e235.

24 Leung KN, Vallero RO, DuBose AJ et al: Imprinting regulates mammalian snoRNAencoding chromatin decondensation and neuronal nucleolar size. Hum Mol Genet 2009; 18: 4227-4238.

Supplementary Information accompanies the paper on European Journal of Human Genetics website (http://www.nature.com/ejhg) 